\title{
A wall-function approach to incorporating Knudsen-layer effects in gas micro flow simulations
}

\author{
D. A. Lockerby ${ }^{1}$, J. M. Reese ${ }^{2}$ and M. A. Gallis ${ }^{3}$ \\ ${ }^{I}$ Department of Mechanical Engineering, King's College London, London WC2R 2LS, UK \\ ${ }^{2}$ Department of Mechanical Engineering, University of Strathclyde, Glasgow G1 1XJ, UK \\ ${ }^{3}$ Engineering Sciences Center, Sandia National Laboratories, PO Box 5800, Albuquerque, NM 87185-0827, USA
}

\begin{abstract}
For gas flows in microfluidic configurations, the Knudsen layer close to the wall can comprise a substantial part of the entire flowfield and has a major effect on quantities such as the mass flow rate through micro devices. The Knudsen layer itself is characterized by a highly nonlinear relationship between the viscous stress and the strain rate of the gas, so even if the Navier-Stokes equations can be used to describe the core gas flow they are certainly inappropriate for the Knudsen layer itself. In this paper we propose a "wall-function" model for the stress/strain rate relations in the Knudsen layer. The constitutive structure of the Knudsen layer has been derived from results from kinetic theory for isothermal shear flow over a planar surface. We investigate the ability of this simplified model to predict Knudsen-layer effects in a variety of configurations. We further propose a semi-empirical Knudsen-number correction to this wall function, based on high-accuracy DSMC results, to extend the predictive capabilities of the model to greater degrees of rarefaction.
\end{abstract}

\section{THE KNUDSEN LAYER}

A Knudsen layer (or kinetic boundary layer) always exists at the interface between a solid boundary and a moving gas. In Figure 1, the solid line schematically shows the structure of the Knudsen layer within a shear flow bounded by a planar wall. In this region the gas is far from a state of local thermodynamic equilibrium; consequently the Navier-Stokes equations and their associated no-slip boundary condition are inapplicable. For many flow situations the scale of the Knudsen layer (at most a few mean free paths in thickness) is negligible in comparison to the macroscopic length scale of interest. However, when the Knudsen number of the flow is greater than about 0.01 the Knudsen layer starts to impact on the entire flow field. Since at relatively low Knudsen numbers $(0.01<\mathrm{Kn}<0.1)$ the Navier-Stokes equations outside of the Knudsen layer may still be at least approximately valid, slip boundary conditions are often employed to incorporate these Knudsen-layer effects within a conventional fluid dynamic solution.

Fundamentally, the slip boundary condition attempts to predict the actual velocity slip that occurs at the gassurface interface: $u_{s l i p}$ in Figure 1. The famous slip conditions proposed by Maxwell [1] are of this type, as are the slip conditions based on adsorption chemistry proposed by Myong [2]. However, within a Navier-Stokes formulation for the core flow, boundary conditions of this type fail to capture completely the macroscopic impact of the Knudsen layer. The dashed and dotted line in Figure 1 demonstrates why, for a simple shear flow, Navier-Stokes simulations with ordinary slip boundary conditions are inaccurate outside of the Knudsen layer.

As an alternative, more sophisticated boundary conditions, such as those due to Kogan [3] and Cercignani [4], attempt to compensate for the structure of the Knudsen layer by adding a "fictitious" velocity slip to the boundary condition: $u_{\text {slip }}^{*}$ in Figure 1. The additional slip ensures that, at least outside the Knudsen layer, a Navier-Stokes solution can be accurate. The dashed line in Figure 1 demonstrates this principle. (Note: by comparison to kinetic theory and DSMC data it can be shown that Maxwell's boundary condition significantly overestimates the amount of actual velocity slip. Consequently, there is a large amount of fictitious slip incorporated within Maxwell's formulation, and this fortuitously improves its predictive capabilities.)

CP762, Rarefied Gas Dynamics: $24^{\text {th }}$ International Symposium, edited by M. Capitelli (C) 2005 American Institute of Physics 0-7354-0247-7/05/\$22.50 


\section{Report Documentation Page}

Form Approved

OMB No. 0704-0188

Public reporting burden for the collection of information is estimated to average 1 hour per response, including the time for reviewing instructions, searching existing data sources, gathering and maintaining the data needed, and completing and reviewing the collection of information. Send comments regarding this burden estimate or any other aspect of this collection of information,

including suggestions for reducing this burden, to Washington Headquarters Services, Directorate for Information Operations and Reports, 1215 Jefferson Davis Highway, Suite 1204, Arlington

VA 22202-4302. Respondents should be aware that notwithstanding any other provision of law, no person shall be subject to a penalty for failing to comply with a collection of information if it

does not display a currently valid OMB control number.

\begin{tabular}{|c|c|}
\hline $\begin{array}{l}\text { 1. REPORT DATE } \\
\mathbf{1 3 ~ J U L ~} \mathbf{2 0 0 5}\end{array}$ & $\begin{array}{l}\text { 3. DATES COVERED } \\
\text { - }\end{array}$ \\
\hline \multirow{3}{*}{$\begin{array}{l}\text { A wall-function approach to incorporating Knudsen-layer effects in gas } \\
\text { micro flow simulations }\end{array}$} & 5a. CONTRACT NUMBER \\
\hline & 5b. GRANT NUMBER \\
\hline & 5c. PROGRAM ELEMENT NUMBER \\
\hline \multirow[t]{3}{*}{ 6. AUTHOR(S) } & 5d. PROJECT NUMBER \\
\hline & 5e. TASK NUMBER \\
\hline & 5f. WORK UNIT NUMBER \\
\hline $\begin{array}{l}\text { 7. PERFORMING ORGANIZATION NAME(S) AND ADDRESS(ES) } \\
\text { Department of Mechanical Engineering, Kings College London, London } \\
\text { WC2R 2LS, UK }\end{array}$ & $\begin{array}{l}\text { 8. PERFORMING ORGANIZATION } \\
\text { REPORT NUMBER }\end{array}$ \\
\hline \multirow[t]{2}{*}{ 9. SPONSORING/MONITORING AGENCY NAME(S) AND ADDRESS(ES) } & 10. SPONSOR/MONITOR'S ACRONYM(S) \\
\hline & $\begin{array}{l}\text { 11. SPONSOR/MONITOR'S REPORT } \\
\text { NUMBER(S) }\end{array}$ \\
\hline
\end{tabular}

12. DISTRIBUTION/AVAILABILITY STATEMENT

Approved for public release, distribution unlimited

13. SUPPLEMENTARY NOTES

See also ADM001792, International Symposium on Rarefied Gas Dynamics (24th) Held in Monopoli (Bari), Italy on 10-16 July 2004.

14. ABSTRACT

15. SUBJECT TERMS

16. SECURITY CLASSIFICATION OF:

a. REPORT unclassified

\section{b. ABSTRACT}

unclassified
17. LIMITATION OF ABSTRACT

$\mathbf{U U}$
18. NUMBER

OF PAGES

6 19a. NAME OF

RESPONSIBLE PERSON unclassified 
As the Knudsen number increases the Knudsen layer becomes a significant proportion of the flow field. This means, for fictitious slip solutions, a large portion of the flow solution is also fictitious. For example, in micro channel simulations fictitious slip conditions lead to a significant over prediction of the mass flow rate [5].

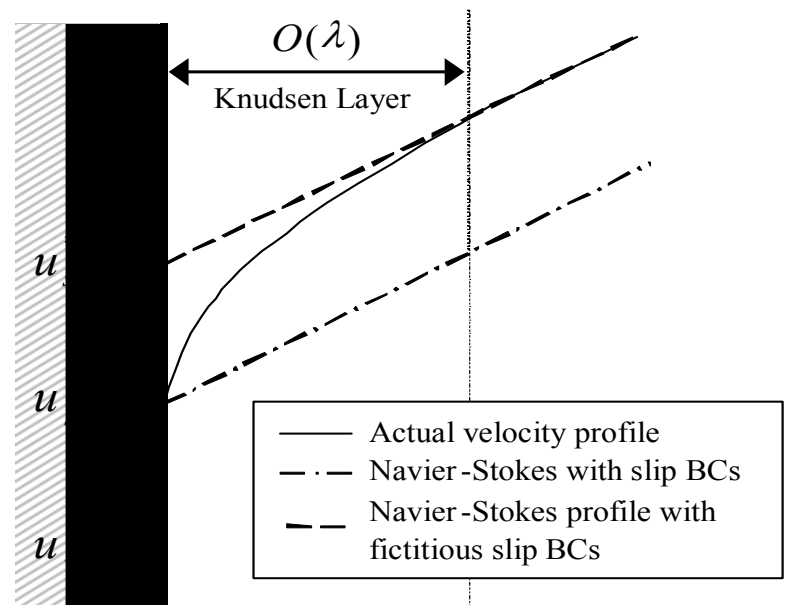

FIGURE 1. Schematic of the structure of the Knudsen layer near a wall, with a comparison of the two types of slip boundary condition.

\section{A WALL-FUNCTION APPROACH}

In this paper we propose a technique for incorporating the effects of the Knudsen layer within the Navier-Stokes constitutive relations, for simulations using the actual, rather than fictitious, velocity slip conditions. We adopt a "wall-function" approach, akin to that used in turbulent boundary layer modelling, and use a solution to the linearised Boltzmann equation for a wall-bounded shear flow to construct a scaling law for the effective viscosity as a function of wall distance. The Knudsen layer also has an effect on the relationship between heat flux and temperature gradient, but here we will restrict our attention to its effect on the stress/strain relationship.

For a planar wall bounding a monatomic gas flow, Cercignani derived the form of the velocity profile, $u$, in the Knudsen layer from the linearised Boltzmann equation [4] and found

$$
u=-\frac{\tau}{\mu}(x+\zeta-\lambda I(x / \lambda))
$$

where $x$ is the normal distance from the planar wall, $\tau$ is the shear stress, $\mu$ is the viscosity, $\lambda$ is the mean free path, and $\zeta$ is a constant. Here we propose a curve-fitted approximation to the velocity correction function, $I(x / \lambda)$ (obtained numerically by Cercignani) as follows:

$$
I(x / \lambda) \approx \frac{7}{20}\left(1+\frac{x}{\lambda}\right)^{-2}
$$

with a mean free path given by

$$
\lambda=\mu \sqrt{\frac{\pi}{2 p \rho}}
$$

where $\rho$ is the density and $p$ is the pressure.

By differentiating Equation (1), a correction to the constitutive relations of the Navier-Stokes equations appropriate in the Knudsen layer can therefore be obtained: 


$$
\tau=-\mu \frac{\mathrm{du}}{\mathrm{d} x} \Psi(x / \lambda)^{-1}
$$

where the "wall-function", $\Psi(x / \lambda)$, is given by

$$
\begin{aligned}
\Psi(x / \lambda) & =1-\lambda \frac{\mathrm{d} I(x / \lambda)}{\mathrm{dx}} \\
& \approx 1+\frac{7}{10}\left(1+\frac{x}{\lambda}\right)^{-3}
\end{aligned}
$$

which, for a given rate of strain, represents a departure of some $40 \%$ from the Navier-Stokes predictions of shear stress at the wall, decreasing to roughly $10 \%$ difference at a distance of one mean free path from the wall.

Since this wall-function is based on Cercignani's linearised Boltzmann solution over a planar surface, our model is strictly only applicable to low-Mach and low-Knudsen number flows over planar surfaces. However, the main heuristic premise of this work is that this wall-function, which is based on these assumptions, can be used as a practical means of giving improved predictions in general geometries and for compressible as well as incompressible flow conditions. This is no less, and no more, justifiable than using fictitious boundary conditions in situations other than those for which they were originally derived. We also assume that the wall function acts as a scaling parameter for viscosity, and consequently affects normal stress similarly to shear stress. This also makes the method easily applicable to other forms of constitutive relation.

The wall-function given in equation (4) may be used in conjunction with Maxwell's general slip condition:

$$
u_{\text {slip }}=-A_{1} \frac{2-\sigma}{\sigma} \lambda \frac{\tau}{\mu}
$$

where $A_{1}$ is the slip coefficient and $\sigma$ is the momentum accommodation coefficient (equal to one for perfectly diffuse molecular deflection and equal to zero for purely specular deflection). As mentioned earlier, in Maxwell's derivation $A_{1}=1$, which is a significant overestimation. Here we use a value obtained from the analytical solution of the linearised Boltzmann equation [4]

$$
A_{1}=\sqrt{\frac{2}{\pi}} \approx 0.8
$$

\section{Planar Couette and Poiseuille Flow Simulations}

To test this proposed wall-function approach, we use a simple centered finite-difference numerical scheme to solve the Navier-Stokes equations for monatomic gas flow in benchmark one-dimensional planar Couette and Poiseuille systems. Figures 2 and 3 show a comparison of solutions obtained using the DSMC method and the Navier-Stokes equations using both the conventional fictitious slip boundary conditions and the wall function approach described above. In both cases the Knudsen number is defined as the ratio of the mean free path to half the channel height $(L)$. Although the differences are fairly minor for these particular benchmarks, results over a range of Mach numbers (M) show that the Knudsen-layer wall function approach produces better agreement with the DSMC data than do conventional fictitious slip solutions.

\section{A SECOND-ORDER KNUDSEN-LAYER CORRECTION}

For flows with a higher Knudsen number, it is expected that the structure and extent of the Knudsen layer changes. In fact, close inspection of Figures 2 and 3 reveals that the wall function solution and the DSMC data do not agree so well at higher Knudsen numbers. As a further (empirical) correction, based on these and other DSMC simulation results, we propose a simple Knudsen-number dependent correction of the function $I(x / \lambda)$, as follows:

$$
I_{2}(x / \lambda)=(1+a \mathrm{Kn}) I(x / \lambda)
$$


where $a$ is a constant chosen to give best agreement with the DSMC simulations of Figure 3 and others; we have found $a \approx 5 / 2$. The second-order wall function that this produces is as follows

$$
\Psi_{2}(x / \lambda)=\Psi(x / \lambda)+\frac{5}{2} \operatorname{Kn}[\Psi(x / \lambda)-1]
$$

Figure 4 shows two high-Kn Poiseuille simulations comparing the DSMC data with Navier-Stokes solutions. Second-order slip boundary conditions [5] are compared to our second-order wall-function with Maxwellian slip conditions; the latter produces much closer agreement with the DSMC data (although it should be noted that at such high Knudsen numbers the Navier-Stokes equations themselves are generally accepted as inappropriate models for the flow). In Figure 4(b) the first-order wall function Knudsen layer, which unlike the second-order wall function has no empirically-tuned parameter, is also shown to give a significantly better prediction than the second-order slip boundary conditions.

\section{Flow Around an Unconfined Sphere}

In this section we investigate low-Reynolds number (monatomic gas) flow past an unconfined sphere for various Knudsen numbers. Figure 5 shows a comparison of the total drag (normalized by the Stokes' drag - the limiting case as the Knudsen number tends to zero) of Milikan's experimentally-fitted curve for air [6] with the Navier-Stokes equations with a Knudsen-layer wall function, the Navier-Stokes equations with a second-order Knudsen-layer wall function, and Basset's conventional slip-flow analysis [7]. The Navier-Stokes equations have been solved by a Runge-Kutta shooting method.

The wall function method gives an improved prediction of drag compared to conventional slip analysis up to Kn $\approx 0.1$. The second-order corrected wall function extends the applicability of the Navier-Stokes equations in this case to even higher Knudsen numbers, $\mathrm{Kn} \approx 0.3$.

\section{SUMMARY}

In this paper we have proposed a wall function approach to capturing Knudsen-layer effects within NavierStokes micro flow simulations. The form of this wall function is based on a curve-fit to an independent numerical solution of the linearised Boltzmann equation for isothermal monatomic planar shear flow. A simple semi-empirical correction to this function has been chosen, based on DSMC data, to extend the predictive capabilities of this method to higher Knudsen numbers. This heuristic method is proposed as a practical means for obtaining improved results for general microfluidic geometries, and for incompressible and compressible flows. The limited test cases we have investigated (planar Couette flow, planar Poiseuille flow, and low-Reynolds number flow around an unconfined sphere) have indicated that our approach is both effective and practical.

At present, we have only considered the Knudsen layer effect on the stress-strain relations of the constitutive relations and not its effect on the heat flux. However, the scaling of effective viscosity described in this paper generates an effective thermal conductivity and, consequently, the relationship between temperature gradient and heat-flux is altered. Whether this models the thermal Knudsen layer correctly will be the subject of future investigations.

\section{ACKNOWLEDGMENTS}

DAL and JMR are grateful to the UK's Leverhulme Trust (Research Project Grant No. F/07040/G) for supporting this research financially, and to Dr David Emerson and Dr Robert Barber of Daresbury Laboratory, UK, for very helpful discussions. MAG performed his work at Sandia National Laboratories. Sandia is a multiprogram laboratory operated by Sandia Corporation, a Lockheed Martin Company, for the United States Department of Energy's National Nuclear Security Administration under contract DE-AC04-94AL85000. 


\section{REFERENCES}

1. Maxwell, J. C., Phil. Trans. Roy. Soc. 170, 231-256 (1879).

2. Myong, R. S., Phys. Fluids 16, 1004-117 (2004).

3. Kogan, M. N., Rarefied gas dynamics, Plenum Press, New York, 1969, pp 367-399.

4. Cercignani, C., Mathematical Methods in Kinetic Theory, Plenum Press, New York, 1990, pp. $232-243$.

5. Hadjiconstantinou, N. G., Phys. Fluids 15, 2352-2354 (2003).

6. Allen, M. D., and Raabe, O. G., J. Aerosol Sci. 13, 537-547 (1982)

7. Basset, A. B., A Treatise on Hydrodynamics, Cambridge University Press, Cambridge, 1888.

a)

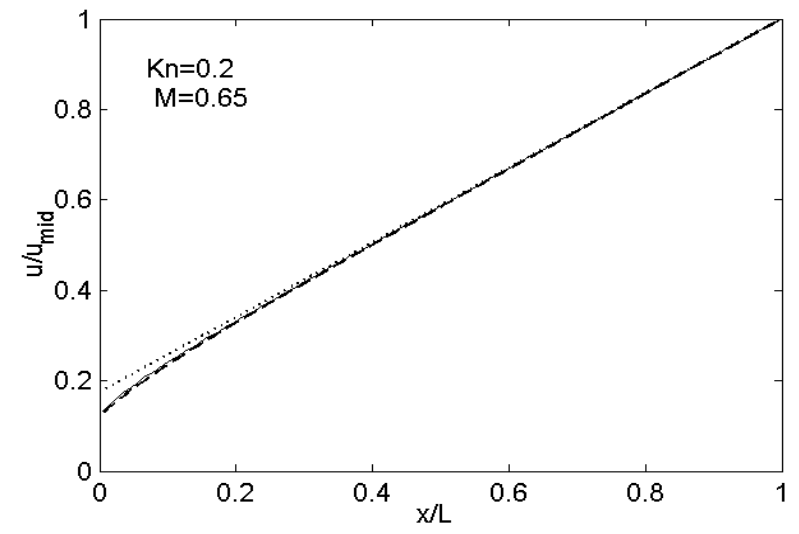

b)

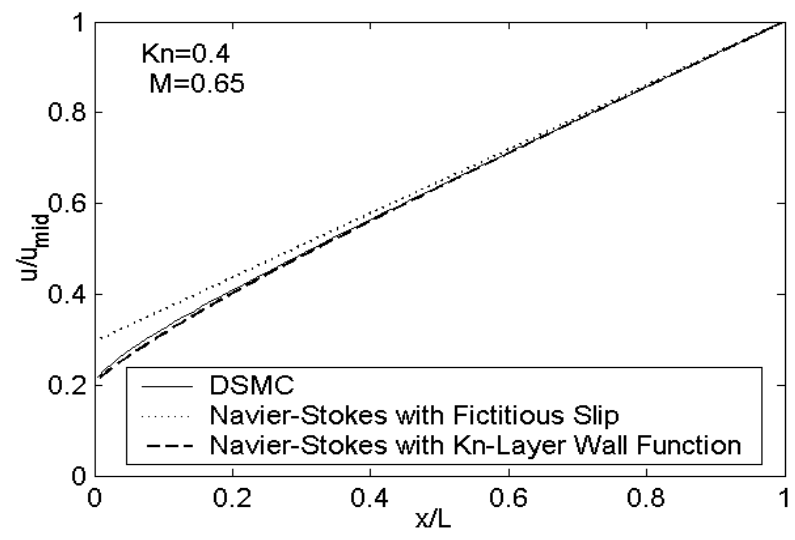

FIGURE 2. Normalised velocity profiles in rarefied Couette flow. DSMC results (-), Navier-Stokes solution with fictitious slip $(\cdots)$, Navier-Stokes solution with Maxwellian slip and the Knudsen-layer wall function $(--)$. The wall at $x / L=0$ is stationary and that at $x / L=2$ is moving at $M=0.65$ with Knudsen numbers (a) $\mathrm{Kn}=0.2$ and (b) $\mathrm{Kn}=0.4$.

a)

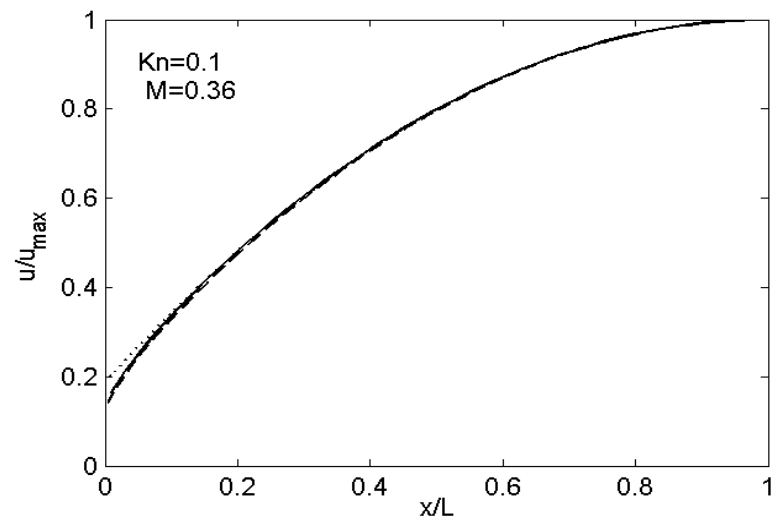

b)

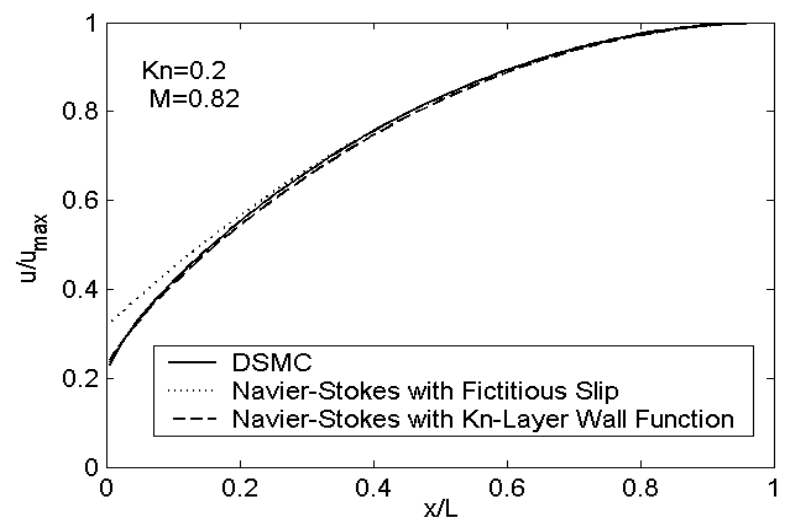

FIGURE 3. Normalized velocity profiles in rarefied Poiseuille flow. DSMC results (-), Navier-Stokes solution with fictitious slip ( $\cdots$ ), Navier-Stokes solution with Maxwellian slip and the Knudsen-layer wall function (- -): (a) $\mathrm{Kn}=0.1, \mathrm{M}=0.36$; (b) $\mathrm{Kn}=0.2, \mathrm{M}=0.82$. 
a)

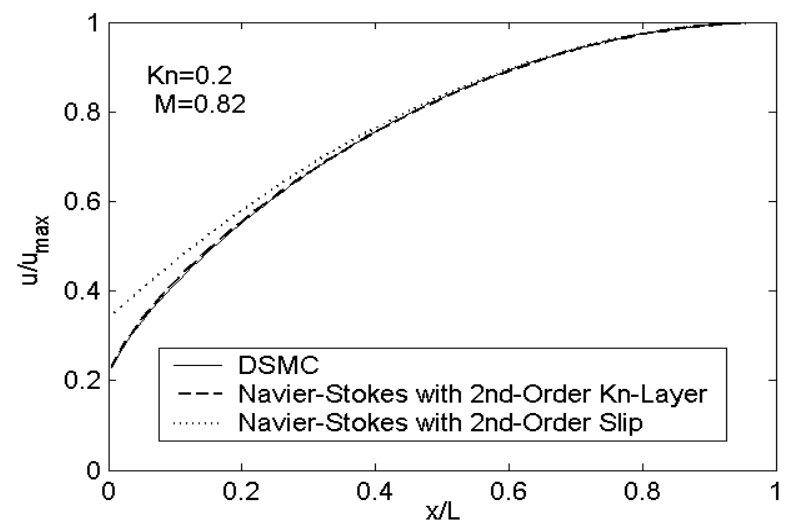

b)

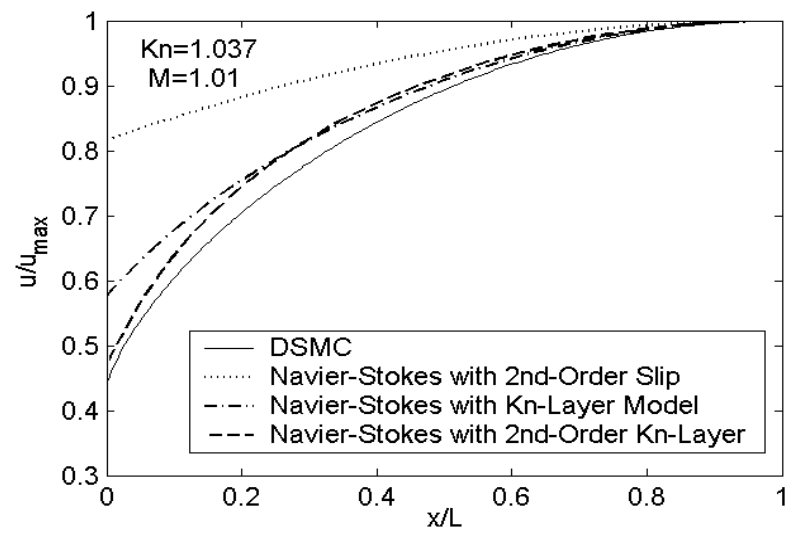

FIGURE 4. Normalized velocity profiles in rarefied Poiseuille flow. DSMC results (-), Navier-Stokes solution with secondorder fictitious slip [5] (*), Navier-Stokes solution with Maxwellian slip and the Knudsen-layer wall function (- $)$ ), NavierStokes solution with Maxwellian slip and the second-order Knudsen-layer wall function (- -): (a) $\mathrm{Kn}=0.2$, M=0.82; (b) $\mathrm{Kn}=1.06, \mathrm{M}=1.01$.

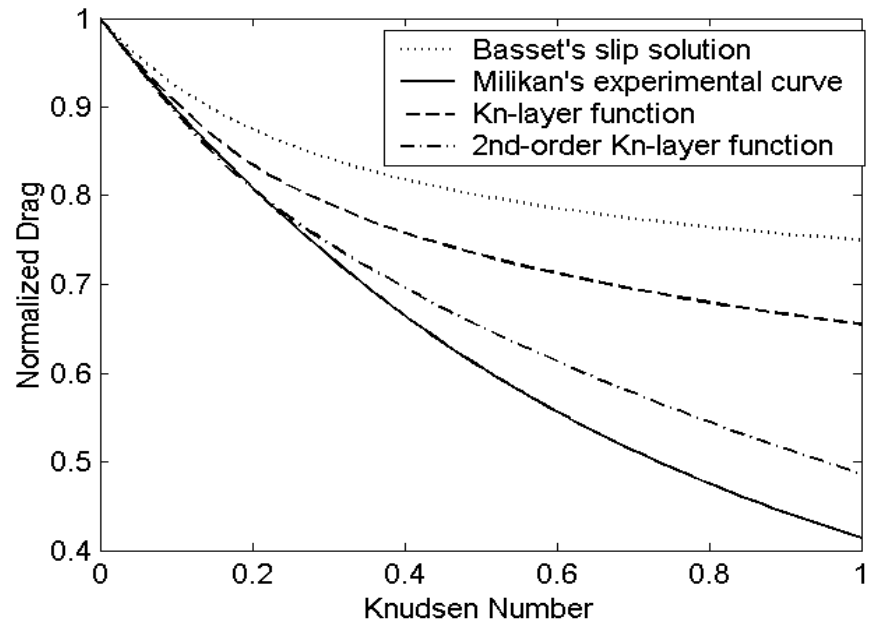

FIGURE 5. Normalized drag over an unconfined sphere over a range of Knudsen numbers. Experimentally-fitted curve [6] (-), Basset's slip solution [7] ( $\cdots)$, Navier-Stokes solution with Maxwellian slip and the Knudsen-layer wall function (--), NavierStokes solution with Maxwellian slip and the second-order Knudsen-layer wall function $(-\cdot)$. [The characteristic length used to define the Knudsen number is the sphere radius.] 